\title{
Heat Absorption by a Thermal System
}

\author{
Zdzisław Pluta, Tadeusz Hryniewicz* \\ Koszalin University of Technology, Raclawicka 15-17, PL 75-620 Koszalin, Poland \\ *E-mail address: Tadeusz.Hryniewicz@tu.koszalin.pl
}

„Rem tene, verba sequentur"

Katon the Elder

\begin{abstract}
The work deals with revealing the quantum nature of the heat absorption phenomenon by a thermal system, describing the phenomenon with a proper energetic interpretation. At first, the existent description of the heat absorption phenomenon by a system has been described. Then the mechanicalthermal analogy was introduced, using it for a better explanation of the determined thermal notions. A set of characteristics of the heat absorption phenomenon was developed by taking advantage of this analogy. The starting point from the source thermal characteristics, being differential dependence of thermal force on temperature, has been regarded. That made it possible to achieve a detail solution, being the reason model of the described natural system, which is the model having a physical sense.
\end{abstract}

Keywords: Heat; Quantum nature of the reality; Temperature; Specific heat; Heat force; Heat work; Heat potential; Potential field; Temperature constant; Thermal energy

\section{INTRODUCTION}

At the very beginning it is worth notifying that there are many phenomena possessing a quantum character. That means the magnitudes describing them change their values between the neighbouring constant values. These constant values characterize the system states where the determined phenomenon occurs. Thus non-continuous, quantum change of the state values takes place, and the continuity of changes touches only the interstate values of the magnitudes describing the studied phenomenon.

It results that one should clearly indicate the appearing layers and surfaces of the natural system. These layers are the determined interstate spaces, and the surfaces are the potential fields. In the spaces everything undergoes changes but on the fields the variability does not occur. One should consider them also on the description plane of natural phenomena. That is a pity, the superficial, simplified, linearized descriptions of the natural systems are usually presented, thus disregarding their true, quantum nature.

One of such phenomena of the quantum nature is the phenomenon of heat absorption by a thermal system. Unfortunately, until now it has not been described adequately. The existent descriptions are so simplified that they made it impossible to recognize the quantum nature of the phenomenon. This is why there is no mentioning about the existence of heat energy, and instead the heat is defined as the energy. 
That work is to explain the quantum nature of the heat absorption phenomenon by the thermal system; it adequately describes that phenomenon, by providing with it a proper, energetic interpretation. Heat and thermal energy are here clearly differentiated.

All that results from the source starting point, the key link of the action chain. This link forms a general, differential record of the phenomena occurring at the same time during the heat exchange in a thermal system.

\section{EXISTENT DESCRIPTION OF THE HEAT ABSORPTION PHENOMENON BY A SYSTEM}

The existent description of this phenomenon should be begun from the literature, on upto-date explanation of the heat magnitude absorbed by the system. That heat has not been defined univocally and clearly. For instance, the literature [1] provides the definition of heat which is the energy that the system of higher temperature transfers to the system of lower temperature, through the other one being in contact, in the process of getting/approaching of both systems to a thermal equilibrium. According to such an interpretation of heat (identified with the energy), that energy varies in a continuous manner. No surprising then that the quantum form of the phenomenon has not been noticed.

Literature [2] also states that heat is the energy. The work [3] discusses the heat exchange. It interprets this as the phenomenon appearing in case if the temperature difference inside a system or between some systems, being in a position able to react one on each other, may occur. However, it does not define the heat.

In the work [4], there are several different explanations of the heat notion. Moreover, in the past there was a hypothesis that heat is a sort of weightless substance. Anton Laurent Lavoisier (1789) called it the calorium. Julius Robert Mayer (1842) was probably to prove that the heat is also a sort of energy. In turn, Helmholtz called the heat of such a part of energy, which may be transferred from one body to another thanks to the temperature difference of both bodies.

One may note, there is no agreement in the opinions regarding the content of the heat notion. The presented standpoints are not uniform, but divergent. In practice it appears, none of the explanations has an adequate character.

The total heat absorbed by a body is determined by the following formula:

$$
Q_{c}=\left.m c\right|_{t_{1}} ^{t_{2}}\left(t_{2}-t_{1}\right)
$$

where: $m$ - body mass, $\left(t_{2}-t_{1}\right)$ - temperature increment, that is the difference between the final $t_{2}$ and initial $t_{1}$ temperature of the body.

In reference to the amount of substance, the equation (1) assumes the form

$$
q_{c}=\frac{Q_{c}}{m}=\left.c\right|_{t_{1}} ^{t_{2}}\left(t_{2}-t_{1}\right)
$$

where the symbol $q_{c}$ denotes the unit heat. That way the magnitude is called in the literature covering thermodynamics [4], as well as that connected with heat exchange [3]. The magnitude 


$$
\left.c\right|_{t_{1}} ^{t_{2}}=\frac{Q_{c}}{m} \cdot \frac{1}{t_{2}-t_{1}}=\frac{q_{c}}{t_{2}-t_{1}}
$$

has been called the average specific heat which absorbs the delivered heat.

In accordance with the literature [6] the specific heat established for the infinitively small the temperature increment $d t$ has been called the real specific heat (real heat capacity):

$$
c=\frac{d q_{c}}{d t}
$$

Further considerations cover the conditions determining the unit heat $q_{c}$. For instance, the references $[4,6]$ mention, that if the dependence $c=c(t)$ is given, then the heat could be determined from the equation

$$
q_{c}=\int_{t_{1}}^{t_{2}} c(t) d t
$$

On the other hand, if the dependence of specific heat on temperature was given, for instance in the form of a function

$$
c(t)=c_{0}+\alpha t+\beta t^{2}
$$

then

$$
q_{c}=c_{0}\left(t_{2}-t_{1}\right)+\frac{\alpha}{2}\left(t_{2}^{2}-t_{1}^{2}\right)+\frac{\beta}{3}\left(t_{2}^{3}-t_{1}^{3}\right)
$$

Next, if the dependence $c(t)$ is known in the form of a plot, the heat $q_{c}$ is determined by the planimetry method of the field (Fig. 1). 


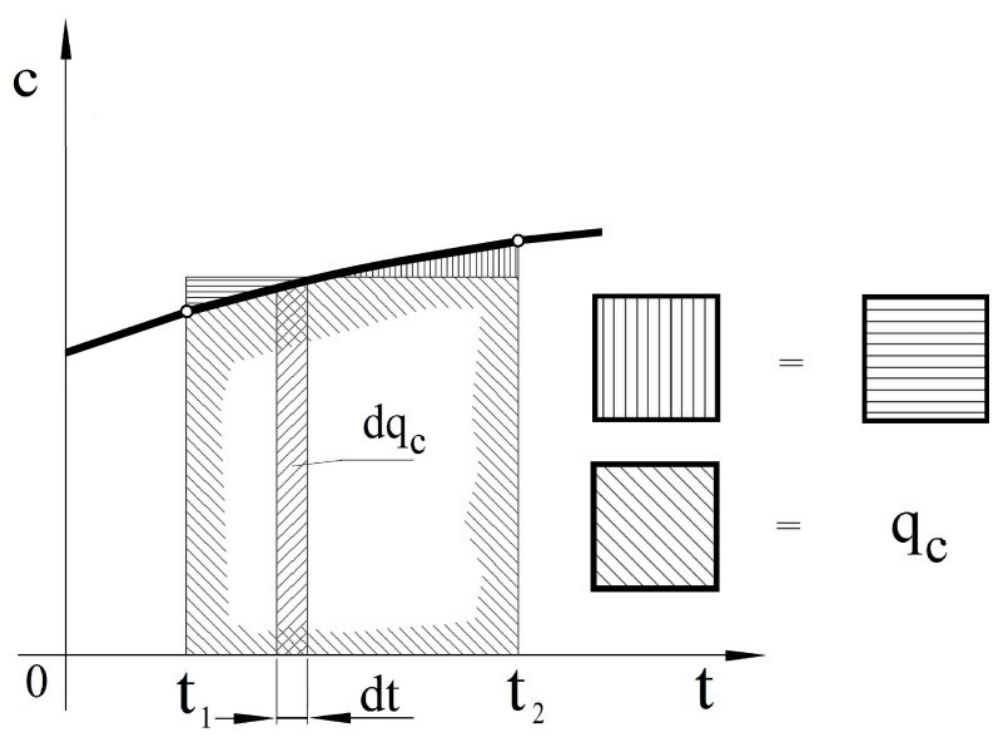

Fig. 1. Heat absorbed $q_{c}$ in the system: $c$ - specific heat, $t$-temperature.

The tables of average specific heat are done usually under foundation of a constant bottom temperature value, e.g. $t_{1}=0{ }^{\circ} \mathrm{C}$. To calculate the absorbed heat by a body in the temperature range of $t_{1} \neq 0$, to $t_{2}$, by means of such given the average specific heat, one should use the formula

$$
q_{c}=\left.c\right|_{0} ^{t_{2}} t_{2}-\left.c\right|_{0} ^{t_{1}} t_{1}
$$

By comparison of the dependences (2), (5), and (8), one obtains the following formula:

$$
\left.c\right|_{t_{1}} ^{t_{2}}=\frac{\int_{t_{1}}^{t_{2}} c(t) d t}{t_{2}-t_{1}}=\frac{\left.c\right|_{0} ^{t_{2}} t_{2}-\left.c\right|_{0} ^{t_{1}} t_{1}}{t_{2}-t_{1}}
$$

As it results from the presented description of the phenomenon of heat absorption by a system, that description is too simplified, and introduced herewith the magnitudes are somewhat doubtful. It will be explained on the background of the presented further the adequate description of the heat absorptions and at the same time transferring the heat by the system bodies. Thus the quest of using the mechanical-thermal analogy will be raised. It will allow to clarify the meaning of thermal magnitudes, with the heat as the problematic subject.

\section{ADVANTAGE OF MECHANICAL-THERMAL ANALOGY}

As it was proved in the former section of the work, the existent definitions of heat have no adequate character. It appears, the undertaken trials to explain the subject notion have not allowed to solve that problem/cognitive quest. Furthermore the heat is something which 
penetrates/permeates, passes, traverses, flows, radiates, rises (convection), all in the medium where the temperature difference occurs. On the grounds of science on the heat, there is a cognitive gap which should be necessarily filled up with the adequate content of the notion of this magnitude.

Therefore, the heat is the physical magnitude as it may be measured and provided with the value. For the time being, that is the way it is characterized. It has been also established, that the heat is the way of the energy transfer. All it is about the heat energy. Thus there is the notion of thermal energy, and heat. They are two different notions and none a proper definition. They are identified, used interchangeably, that leads to the cognitive pandemonium, if it refers to the determined fragments of the natural reality.

In science, there is the equilibrium of mechanical work and heat, indicated by a known experiment of Joule (1843). The relationship of heat on the mechanical work is regarded by the thermal equivalent of mechanical work, and the dependence of the mechanical work on heat is covered by the mechanical equivalent of heat. Here also, the heat notion is not explained. The heat is still the non-determined notion.

The heat notion may be explained on the way of reasoning. However, in this case the cognitive fundamentals, using the philosophic approach and principal methods of the cognitive work, should be considered. In this case the method of mechanical-thermal analogy, resulting from the identity of mathematic form of equations describing the mechanical work and heat, should be applied.

Unfortunately, that analogy has not been noticed as yet, even if the analogy is used, concerning the problems connected with the heat exchange. For instance, the work [3] turns the attention on the analogy occurring between the heat exchange and the momentum exchange, by treating it as the hydromechanic-thermal analogy. The works [7-10], in turn, explain the use methods of electrical analogy to the studies of flow in the complex elements of the fluid-flow machines, in this also the ventilators.

At the very beginning of the necessary considerations, concerning the mentioned above mechanical-thermal analogy, one should state that in fact it is about the thermal work. That is the source of all other investigations. Coming out of this initial point, one may derive and explain the notions of the thermal force, thermal power, and thermal potential. The equivalents are the following notions: mechanical work, mechanical force, mechanical power, and the mechanical potential.

\section{MECHANICAL ESSENCE OF THE WORK, FORCE, POWER, AND POTENTIAL}

The mechanical work is the work of solid, or the body being in the solid state of aggregation. That general qualitative definition was formed in accordance with the rule of systematics/satiety [5], which determines the method of creation of all definitions. It is about the two-order systematics ( 1 - work, 2 - mechanical work).

That general qualitative definition may be extended respectively. That extended definition will be the three-order systematics. The third its order forms the closer explanation which says that the work forms the connection between the reason and effect. Therefore that three-order systematics has the following configuration: 1 - work, 2 - mechanical work, 3 the mechanical work, forming a determined connection between the reason and effect.

Quantitatively, the definition of the mechanical work $L$ says that the physical magnitude is the product of the mechanical work $F$ and the displacement $s$ of a solid. That force is the measure of reason, whereas the displacement (path length) is the measure of effect. Therefore 


$$
L=F \cdot s
$$

and in the elemental form

$$
d L=F(s) \cdot d s
$$

so that

$$
L=\int_{0}^{s_{0}} d L=\int_{0}^{s_{0}} F(s) \cdot d s
$$

Of course, the first form of the formula on work is related to the situation, when the force is constant, whereas the second one, that elemental, regards the variability of that force on the length of the way of displacement or the deformed body. That second one, variable force, may be determined as the unit work, that is

$$
l(s)=\frac{d L}{d s}
$$

The mechanical power $N_{L}$ is the magnitude generally known. It concerns the work variability $L$ in time $\tau$, that is the work flux $\dot{L}$, then

$$
N_{L}=\dot{L}=\frac{d L}{d \tau}
$$

The presented characteristics are concerned with the mechanical behaviours of the material body in the space and interstate space-time, that is the matter occurring between the neighbouring potential fields, where the determined energetic states of the material body take place.

Now there is the need to introduce the magnitude which will characterize these states. That is the energy of the material body which is the ability to perform a work, or the readiness to perform the work over it, at the transition on the neighbouring potential field. The measure of energy is the potential $V_{F}$, being the product of the potential field intensity and the distance $s_{0-1}$ between the neighbouring potential fields. The field intensity is determined by the initial force $F_{0}$. Therefore

$$
V_{F}=F_{0} \cdot s_{0-1}
$$

As it results, the mechanical potential (measure of the mechanical energy) is equivalent with the mechanical work. However, it does not mean that both these magnitudes are analogous. There are some determined connections between them, resulting from the relations 


$$
\begin{gathered}
k_{L}=\frac{L}{V_{F}}=\frac{\int_{0}^{s_{0-1}} F(s) \cdot d s}{F_{0} \cdot s_{0-1}} \\
k_{V}=\frac{V_{F}}{L}=\frac{F_{0} \cdot s_{0-1}}{\int_{0}^{s_{0-1}} F(s) \cdot d s}
\end{gathered}
$$

which the relations are named the equivalents: equivalent of the mechanical work $k_{L}$ and the equivalent of the mechanical potential $k_{V}$, respectively.

The notions of the mechanical magnitudes: work, force, power, are generally known, but the energy (measure of this magnitude, that is the potential) has not been clearly explained as yet. The introduced herewith the definition has the adequate character and fully reveals the essence of this notion. That has been used now, reflected by the following exemplary works [11-17].

\section{THERMAL CHARACTER OF THE WORK, FORCE, POWER, AND POTENTIAL}

The mechanical-thermal analogy allows to determine the heat (thermal work) in the following manner:

$$
d Q=F(t) \cdot d t
$$

where the differential $d Q$ denotes the elemental thermal work, $F(t)$ is the thermal force, and the differential $d t$ is the elemental temperature.

Of course this thermal force is the product of the mass $m$ of a material body (thermodynamic factor) and its specific heat $c(t)$, then

$$
d Q=m \cdot c(t) \cdot d t
$$

so further

$$
Q=\int_{t_{0}}^{t_{1}} F(t) \cdot d t=m \int_{t_{0}}^{t_{1}} c(t) \cdot d t
$$

where $t_{0}$ is the initial temperature, $t_{1}$ the final temperature. 
In analogy to the previous action, this force is a unit work, that is $F(t)=m \cdot c(t)$. Therefore that unit thermal work takes the following form

$$
q(t)=\frac{d Q}{d t}
$$

The thermal power $N_{Q}$ is the work $Q$ variability, that results from the temperature $t$ change. This is now the heat flux (of thermal work), so

$$
N_{Q}=\dot{Q}=\frac{d Q}{d t}
$$

It is time now to explain the notion of thermal energy. First, however, it should be noted that until now there is no clear univocal definition of that magnitude. Thus let us focus on the work [18], by J. Miquel Rubi. Therein he states that the thermodynamics is one of such a part of physics which is understood erroneously. There are many notions in use by different scientists which do not necessarily reflect the exact meaning and the relevant subtleties. Thus the author of [18] has mentioned that he touches deep into the theory but expressed his feeling to be careful in this matter.

How then J. Miquel Rubi understands the energy notion? It is reflected with his explanation of the phenomenon of thermal equilibrium: ,, While bringing into contact two bodies of different temperature, the energy in the form of heat flows from the warmer to cooler one. It lasts until the equilibrium of temperatures of both bodies is achieved, which is the thermal equilibrium".

The presented explanation also does not reveal the true essence of energy. Energy does not flow and is not a form of heat. They are two quite different magnitudes. These basic differences are distinctive at the initial stage of their characterization, if the kind of magnitudes is clarified.

The energy is a mental magnitude, the mind creature, the notion category. Therefore it is not a physical magnitude, which could be measured and recorded by means of mathematic tools, to determine its value, price or consumption. One may differentiate the sort of energy but not its form. All it is about the thermal energy. The heat, or rather thermal work, is the way of energy transfer, and this transfer has non-continuous, jump, quantum character.

Thus the energy is the function of state. Therefore its presence is detected on the determined energetic levels only (potential fields). Between these levels/fields, there is no energy at all. There the intensified temperature variability occurs, proving of that the thermal work takes place.

Of course, now a determined measure, as the physical magnitude to be undergoing measurements, should be assigned to the energy (mental magnitude). There is such a measure. That is a potential, being the product of the potential field intensity and a determined space (or space-time) resistance, dividing the neighbouring potential fields.

One may now present the adequate definition of the thermal energy. The thermal energy of a material body (of thermodynamic agent) is its ability to perform the work, or the readiness to perform the work over it, at the transition onto the neighbouring potential field.

The measure of this energy is the potential $V_{F}$, being the product of the potential field intensity and the thermal distance between these fields. The field intensity is the initial thermal force $F_{0}$ that is the initial unit thermal work, denoted as $q_{0}$. The thermal distance is 
the difference $t_{1}-t_{0}$ of temperatures, which determine the position of the neighbouring potential fields. That refers to the limits of the definite integral, determining the thermal work. Therefore

$$
V_{F}=F_{0}\left(t_{1}-t_{0}\right)=m c_{0}\left(t_{1}-t_{0}\right)
$$

where $c_{0}$ refers to the initial specific heat of a determined thermodynamic agent.

\section{GENERAL CHARACTERISTICS OF HEAT ABSORPTION BY A THERMAL SYSTEM}

The characteristics is the dependence of thermal force $F$ on temperature $t$, that is $F=f(t)$. That force is the product of the heated body mass and its specific heat; thus $F(t)=m \cdot c(t)$.

However, at first it is worth presenting the most general form of the source differential equation. That form is referred to all phenomena and/or processes, having a quantum course which is changing by a jump. The phenomena are considered in macro-scale. Of course, such a sort of changes is related to the states, not the courses between the states, because between them the continuous changes of a determined magnitude take place.

The general differential equation, excerpted from [19], has the following configuration:

$$
d Z= \pm \frac{\partial Z}{\partial N} d N
$$

where: $d Z$ - total differential of the dependent variable; $d N$ - total differential of the independent variable; $\frac{\partial Z}{\partial N}$ - partial derivative of the dependent variable, referred to the independent variable. The signs $( \pm)$ are the algebraic operators fulfilling a determined role. The sign $(+)$ has a formal meaning, confirming the physical sense of a determined dependence. The sign (-) ascribes such a sense to the determined record.

Considered here the phenomenon of heat absorption describes two magnitudes, namely the thermal force $F$ and temperature $t$. Thus that force will appear as the independent variable, and the temperature will take the role of the independent variable. Regarding the fact that the course of the considered magnitude is exponential and rising degressively, then

$$
d F=-\frac{\partial F}{\partial t} d t
$$

The scheme of creation of the adequate description of the dependence $F=f(t)$ is presented in Fig. 2, revealing all elements of this reasoning process. The curve illustrating that dependence comes out of the initial point , 0 ” of the coordinates $t=t_{0}, F=F_{0}$, and further it has the course rising exponentially and degressively. It completes its non-linear course in the point , $1 "$, where the body heating phenomenon has its end. 




Fig. 2. Indicative course of the dependence of thermal force $F$ on temperature $t$.

Now the integration of dependence (16) on both sides should be done remembering that the total differential is the state function. It is needed to determine the states which are the integration limits of this thermal phenomenon.

The mentioned limits are the potential fields. These fields are situated on two directions; one of them is the direction of thermal force, whereas the other one is the direction of temperature. On the first direction, one may find: the bottom force stable potential field $(B S P F)_{F}$, upper force stable potential field $(T S P F)_{F}$, force unstable potential field $(A P P)_{F}$, and the nominal potential field $N P F$. On the second direction, there are the following fields: the temperature stable potential field $(S P F)_{t}$, and temperature unstable potential field $(A P F)_{t}$. Between the fields $(B S P F)_{F}$ and $(T S P F)_{F}$ there is the potential band (thick dotted area in Fig. 2). The fields $(T S P F)_{F},(A P F)_{F},(S P F)_{t}$ and $(A P F)_{t}$ limit/reduce the proper temperature-force space (sparsely dotted area), where the real exponential and degressively rising changes of the thermal force occur (solid line). Between the fields $(A P F)_{F}$ and NPF 
there is an improper space, fulfilling here an auxiliary role (this is also an auxiliary mathematic tool).

The curve of thermal force, comprised between the points $0-1$, is the envelope of rightangled triangles, moving with its horizontal leg on the nominal potential field $N P F$; with that horizontal leg being invariable and equal to the temperature constant $T$, whereas the vertical leg varies respectively, decreasing within the displacement of the triangle in the temperature direction. That nominal field is situated symmetrically against the level of the heating body phenomenon completion, in the distance from it equal to the length of the real space in the direction of the thermal force.

Thus over the real/proper space there is the above mentioned the improper space situated, where the curve (dashed line) approaches the asymptote, being that nominal potential field. That creature is surely an auxiliary design, needed to the description of the real curve, reflecting the adequate dependence of the thermal force on temperature.

Now one may proceed with the integration of the equation (16). At integrating of that equation one should mark the integrals limits of the total differentials. That means

$$
\int_{F_{0}+\Delta F}^{F_{1}+\left(F_{1}-F_{0}\right)} d F=-\frac{\partial F}{\partial t} \int_{\Delta t}^{\Delta t+T} d t
$$

and further

$$
2\left(F_{1}-F_{0}\right)-\Delta F=-\frac{d F}{d t} T
$$

or

$$
\frac{d F}{2\left(F_{1}-F_{0}\right)-\Delta F}=-\frac{1}{T} d t
$$

One may notice, the partial derivative has been substituted by the quotient of total derivatives. It could be done that way because the total differentials have been clearly determined by introducing the limits of the integrals.

Furthermore, by integrating both sides of the equation (19), one obtains the following result

$$
\ln \left[2\left(F_{1}-F_{0}\right)-\Delta F\right]=-\frac{1}{T} t+C^{*}
$$

that is

$$
2\left(F_{1}-F_{0}\right)-\Delta F=e^{-\frac{t}{T}+C^{*}}=e^{C^{*}} \cdot e^{-\frac{t}{T}}=C e^{-\frac{t}{T}}
$$

After regarding that for $\Delta t=t-t_{0}=0$, that is $t=t_{0}$, the magnitude $\Delta F=0$, one obtains 


$$
C=2\left(F_{1}-F_{0}\right) \cdot e^{\frac{t_{0}}{T}}
$$

and after substituting (22) to (21), and further, after relating to $\Delta F=F-F_{0}$

$$
F=F_{0}+2\left(F_{1}-F_{0}\right) \cdot\left(1-e^{-\frac{t-t_{0}}{T}}\right)
$$

One may now determine the second coordinate of the point „1”, i.e. $(\Delta t)_{0-1}=t_{1}-t_{0}$. That is obtained by introducing $F=F_{1}$ and $t=t_{1}$ to the equation (23). Therefore

$$
(\Delta t)_{0-1}=T \ln 2
$$

\section{ANALYSIS OF ADEQUATE DEPENDENCE OF THERMAL FORCE ON TEMPERATURE}

The dependence (23) presents a final, analytical form of dependence of the thermal force $F$ on temperature $t$. It is also the outcome basic functional thermal characteristics of the heated thermodynamic agent. Based on this, now further derivative functional characteristics may be created. Of course this outcome characteristics contain the determined parametric characteristics, i.e. $F_{0}, F_{1}, t_{0}$, and $T$.

The first derivative functional characteristics is the dependence of the increment rate (tempo, intensity) of thermal force on temperature. Thus

$$
k_{F}=\frac{d F}{d t}=\frac{2\left(F_{1}-F_{0}\right)}{T} e^{-\frac{t-t_{0}}{T}}=k_{F}^{0} \cdot e^{-\frac{t-t_{0}}{T}}
$$

where $k_{F}^{0}$ is the initial rate of the force increment. For $t=t_{0}$ that rate $k_{F}=k_{F}^{0}$, and for $t=t_{1}$, i.e. $(\Delta t)_{0-1}=t_{1}-t_{0}=T \ln 2$, the rate $k_{F}=0.5 k_{F}^{0}$.

The course of this magnitude is illustrated in Fig. 3. 


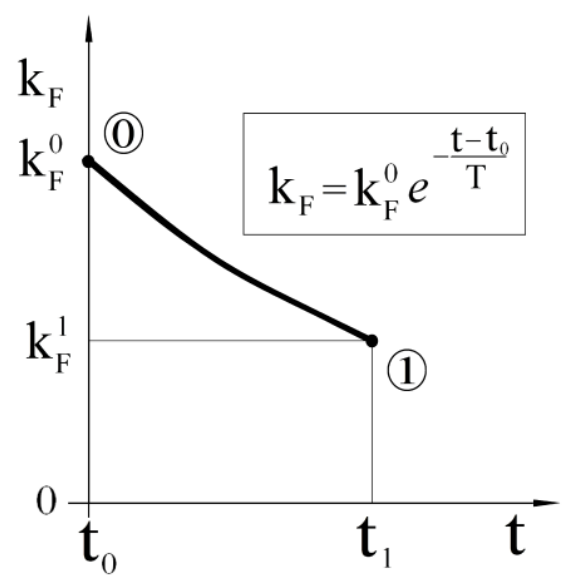

Fig. 3. Dependence of the increment rate of thermal force on temperature.

The graphic image (Fig. 4 a) of the initial dependence, that is (23), may be the basis to explain the essence of further derivative magnitudes, that is the thermal work (Fig. 4 b) and initial (Fig. $4 \mathrm{c}$ ) and final thermal potential (Fig. 4d). These potentials are the measures of thermal energies: initial, and final.

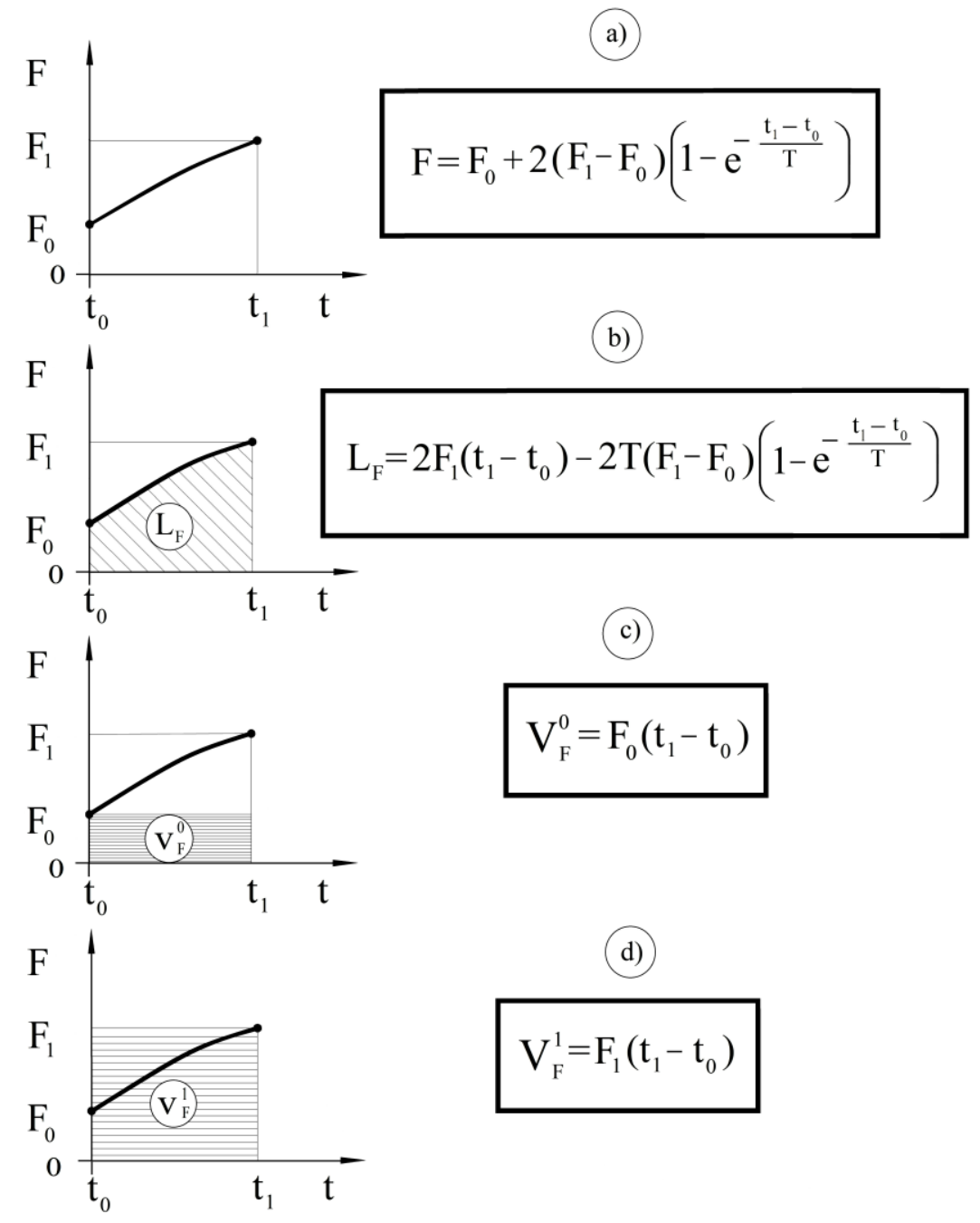

Fig. 4. Illustration of the dependence of force (a), work (b), initial (c), and final (d) thermal potential on temperature. 
The thermal work $L_{F}$ corresponds with the field under the curve $L_{F}=f(t)$, described by the formula of the following form:

$$
\begin{gathered}
L_{F}=\int_{t_{0}}^{t_{1}} F(t) \cdot d t=\int_{t_{0}}^{t_{1}}\left[F_{0}+2\left(F_{1}-F_{0}\right) \cdot\left(1-e^{\left.-\frac{t-t_{0}}{T}\right)}\right) \cdot d t=\right. \\
=2 F_{1}\left(t_{1}-t_{0}\right)-2 T\left(F_{1}-F_{0}\right) \cdot\left(1-e^{-\frac{t_{1}-t_{0}}{T}}\right)
\end{gathered}
$$

The mentioned thermal potentials (initial $V_{F}^{0}$ and final $V_{F}^{1}$ ) are described by the following formulae:

$$
\begin{aligned}
& V_{F}^{0}=F_{0}\left(t_{1}-t_{0}\right) \\
& V_{F}^{1}=F_{1}\left(t_{1}-t_{0}\right)
\end{aligned}
$$

By regarding further that the thermal force $F$ is the product of the heated body mass and its specific heat, the formulae (23), (25), (26), (27), (28) assume the following configurations:

$$
\begin{gathered}
F=m\left[c_{0}+2\left(c_{1}-c_{0}\right) \cdot\left(1-e^{\left.-\frac{t-t_{0}}{T}\right)}\right]\right. \\
k_{F}=\frac{2 m\left(c_{1}-c_{0}\right)}{T} e^{-\frac{t-t_{0}}{T}}=k_{F}^{0} \cdot e^{-\frac{t-t_{0}}{T}} \\
L_{F}=2 m c_{1}\left(t_{1}-t_{0}\right)-2 m T\left(c_{1}-c_{0}\right) \cdot\left(1-e^{-\frac{t_{1}-t_{0}}{T}}\right) \\
V_{F}^{0}=m c_{0}\left(t_{1}-t_{0}\right) \\
V_{F}^{1}=m c_{1}\left(t_{1}-t_{0}\right)
\end{gathered}
$$

By recording in the formula (29) the force $F$ as a product $m \cdot c$, one obtains as follows: 


$$
m \cdot c=m\left[c_{0}+2\left(c_{1}-c_{0}\right) \cdot\left(1-e^{-\frac{t-t_{0}}{T}}\right)\right]
$$

that means

$$
c=c_{0}+2\left(c_{1}-c_{0}\right) \cdot\left(1-e^{-\frac{t-t_{0}}{T}}\right)
$$

that is the dependence of the specific heat on temperature.

\section{CONCLUSION}

The problem of heat absorption through a thermal system has been considered. Apart from the existent notions ordering, quite new notions have been introduced. It appears, the existent notion of heat was identified as the heat potential being the measure of heat energy. The use of mechanical-thermal analogy made it possible to explain several terminology quests. Thus the set of characteristics of the heat absorption phenomenon consists of: the thermal force, heat work, thermal potential, rate/intensity of increment of thermal force, and the specific heat.

The outcome of source, that is of the starting differential equation, being differential dependence of the thermal force on temperature, made it possible to achieve the analytic, functional characteristics of the title phenomenon. That characteristics, being the causal model of the described natural system, has a deep physical reasoning.

It is worth stating that such an approach, coming out of the source differential equation, has been used by the authors earlier [20,21]. In those cases quite a complex dependence of the tool life under cut on the main velocity in the machining system, was used [20] and the kinetics of tool edge fixed flexibly was solved [21].

\section{REFERENCES}

[1] J. Blinowski, J. Trylski, Physics for candidates for studies, PWN, Warszawa 1973 (in Polish).

[2] M. Jeżewski, Physics, PWN, Warszawa 1966 (in Polish).

[3] B. Staniszewski, Heat exchange. Theoretical foundations, PWN, Warszawa 1980 (in Polish).

[4] S. Ochęduszko, Applied thermodynamics, WNT, Warszawa 1967 (in Polish).

[5] M. Mazur, Technical terminology, WNT, Warszawa 1961 (in Polish).

[6] S. Ochęduszko, J. Szargut, H. Górniak, A. Guzik, S. Wilk, Exercises of technical thermodynamics PWN, Warszawa 1968 (in Polish).

[7] T. Chmielniak, A. Szafraniec, Przeglad Mechaniczny 2 (1977) 41-45 (in Polish). 
[8] M. Hackeschmidt, Strömungstechnik-Ähnlichkeit. Analogie-Modell. Lipsk 1972.

[9] M. Hackeschmidt M., Die Elektroanalogie ein Mittel zur Lösung komplizierter Feldprobleme. Habilitationsschrift, Technische Universität, Dresden 1964/65.

[10] M. Hackeschmidt, Zur Ermittlung der reibungsfreien Strömung durch rotierende Schaufelsterne aus beliebigen Profilen unter Verwendung der Elektroanalogie. Maschinenbautechnik 8 (1963).

[11] Tadeusz Hryniewicz, Zdzisław Pluta, Understanding the Teaching Process or the Lost Essence of Energy. Proc. of the $8^{\text {th }}$ Word Conference on Continuing Engineering Education, Theme A.: Knowledge, Skills and Competency, May 12-16, 2001, Toronto, Ontario, Canada, pp. 25-31.

[12] Zdzisław Pluta, LAB 4 (2005) 43-46 (in Polish).

[13] Zdzisław Pluta, Energetyka 7 (2005) 496-501 (in Polish).

[14] Zdzisław Pluta, Return to the sources, Forum Akademickie 3 (1999) 58-60 (in Polish)

[15] Zdzisław Pluta, Energetic aspects of cutting using single abrasive grain fixed flexibly. Proc. 23rd Sci. School on Abrasive Treatment. Rzeszów-Myczkowice, September 2000, pp. 303-309 (in Polish).

[16] Zdzisław Pluta, Potential and the work of a single abrasive grain, Proc. $25^{\text {th }}$ Sci. School on Abrasive Treatment. Wrocław-Duszniki Zdrój, 11-14 September 2002, pp. 389-394 (in Polish).

[17] Zdzisław Pluta, Determination of energetic potential of geometrically compact abrasive tools, Proc. 25th Sci. School on Abrasive Treatment. Wrocław-Duszniki Zdrój, 11-14 September 2002, pp. 395-400 (in Polish).

[18] J. Miquel Rubi, Świat Nauki (208)12 (2008) 44-49 (in Polish).

[19] Zdzisław Pluta, Fundamentals of surface smoothing by elastic abrasive wheels, Wydawnictwo Politechniki Koszalińskiej, Koszalin 2007 (in Polish).

[20] Zdzisław Pluta, Tadeusz Hryniewicz, Int. J. Adv. Manuf. Technol. 51 (2010) 217-223, DOI:10.1007/s 00170-010-2595-5.

[21] Zdzisław Pluta, Tadeusz Hryniewicz, Int. J. Adv. Manuf. Technol. 62(5) (2012) 529-542, DOI:10.1007/s 00170-011-3813-5. 